\title{
Comparison of long-term clinical outcomes among zotarolimus-, everolimus-, and biolimus-eluting stents in acute myocardial infarction patients with renal impairment
}

\author{
Seok Oh*(-), Dae Young Hyun®, Kyung Hoon Cho®, Ju Han Kim®, Myung Ho Jeong*®(])
}

Department of Cardiology, Chonnam National University Hospital, Gwangju, Korea

\begin{abstract}
Background: It is important to determine the best drug-eluting stent (DES) for acute myocardial infarction (AMI) in patients with renal impairment. In this studythe outcomes of everolimus-eluting stents (EESs), zotarolimus-eluting stents (ZESs) and biolimus-eluting stents (BESs) were evaluated.

Methods: From the Korea Acute Myocardial Infarction-National Institutes of Health registry, a total of 1,470 AMI patients with renal impairment undergoing percutaneous coronary intervention (PCI) were enrolled (816 with EES, 345 with ZES, and 309 with BES). Renal impairment was defined as creatinine clearance $<60 \mathrm{~mL} / \mathrm{min} / 1.73 \mathrm{~m}^{2}$ estimated by the Cockcroft-Gault method. Major adverse cardiac and cerebrovascular events were determined as the composite of all-cause death, non-fatal myocardial infarction (MI), cerebrovascular accident, any revascularization, rehospitalization and stent thrombosis. All clinical outcomes were analyzed.

Results: The baseline characteristics of the patients revealed no significant difference between the three groups, except for Killip classification $>2$, beta-blockers, lesion type, vascular approach, staged PCI, left main coronary artery (LMCA) complex lesions, LMCA PCI, and the number and length of implanted stents. In the Kaplan-Meier analysis, similar clinical outcomes were derived from the unadjusted data between the three DES groups. However, after the inverse probability of treatment weighting, a statistically significant difference was found in non-fatal MI, which implied a higher incidence of non-fatal $M I$ in the ZES group than in the other two DES groups.

Conclusions: In AMI patients with renal impairment, there was no significant difference between the three stent groups in terms of long-term clinical outcomes, except for non-fatal MI. (Cardiol J)
\end{abstract}

Key words: myocardial infarction, renal insufficiency, drug-eluting stents, zotarolimus, everolimus, biolimus

\section{Introduction}

The incidence of acute coronary syndrome (ACS) with concomitant acute myocardial infarction (AMI), is gradually rising, leading to serious socioeconomic problems. Risk factors for coronary artery disease (CAD) such as diabetes, hyperten- sion, and chronic kidney disease (CKD) are similarly increasing. Among these risk factors, CKD is an independent risk factor for cardiovascular disease $[1,2]$. In patients with end-stage kidney disease (ESKD), the incidence of cardiovascular diseases is $8.8-10$ times higher than the general population $[3,4]$.

Address for correspondence: Dr. Myung Ho Jeong, Department of Cardiology, Chonnam National University Hospital, 42, Jebong-ro, Dong-gu, Gwangju 61469, Korea, tel: +82-62-220-6243, fax: +82-62-227-3105, e-mail: myungho@chollian.net Received: 28.04.2021 Accepted: 18.08.2021 Early publication date: 26.08.2021

This article is available in open access under Creative Common Attribution-Non-Commercial-No Derivatives 4.0 International (CC BY-NC-ND 4.0) license, allowing to download articles and share them with others as long as they credit the authors and the publisher, but without permission to change them in any way or use them commercially. 
Acute myocardial infarction, a medical emergency, is a type of ACS that requires rapid revascularization. The advent of coronary stents, utilized for the treatment of coronary stenosis, has contributed to a decrease in both, restenosis and the likelihood of emergency coronary artery bypass grafting (CABG) $[5,6]$. After 2002, the emergence of drug-eluting stents (DESs), including the sirolimus-eluting stents (SESs) and paclitaxeleluting stents significantly reduced the incidence of restenosis and the need for repeat revascularization compared with balloon angioplasty. The use of first-generation DES (1G-DES) has reduced the rate of target lesion revascularization (TLR) and CABG as a treatment option after stent implantation $[7,8]$. However, there is an increasing concern about stent thrombosis, one of the most catastrophic phenomena of percutaneous coronary intervention (PCI), which manifests as ST-segment elevation myocardial infarction (STEMI) and/or sudden cardiac arrest requiring repeat revascularization [9]. Newer generation durable polymer-coated DESs using zotarolimus or everolimus, called second-generation drug-eluting stents (2G-DESs), were developed to ameliorate polymer biocompatibility, leading to a significant reduction in in-stent restenosis, stent thrombosis, the duration of dual antiplatelet therapy (DAPT), and bleeding complications [10, 11]. In addition, biolimus-eluting stents (BESs), which use a biodegradable polymer, have been developed to treat long-term vascular complications related to durable polymers. Studies have shown that BES reduce late stent thrombosis compared with $1 \mathrm{G}$ DES [12]. In addition, it exhibited similar safety and efficacy characteristics compared with those of other 2G-DESs [13, 14].

It has been established that cardiovascular disease is a leading cause of morbidity and mortality among CKD patients. CKD progresses through supply-demand mismatch, ischemic preconditioning, collateralization of blood vessels, and a high prevalence of left ventricular hypertrophy, leading to the development of $\mathrm{CAD}[2,15]$.

In this study, the focus was on the differences in clinical outcomes between DESs in AMI patients. There is a paucity of clinical data on the difference in the outcomes between the three stent groups (zotarolimus-eluting stents [ZESs], everolimus-eluting stents [EESs], and BESs) in patients with AMI and renal impairment (AMI-RI). This clinical study aimed to elucidate the clinical differences between these three types of stents in patients with AMI and concomitant renal impairment undergoing PCI.

\section{Methods}

\section{Study population}

The study population was extracted from the Korea Acute Myocardial Infarction Registry-National Institutes of Health (KAMIR-NIH), a nationwide, multicenter, online observational cohort study. The KAMIR-NIH consecutively enrolled AMI patients at 20 major cardiovascular institutes between 2011 and 2015. Among 13,104 AMI patients, a total of 4,692 AMI-RI patients were initially screened. The exclusion criteria included patients who: (a) had a prior myocardial infarction (MI); (b) died during index hospitalization; (c) underwent no PCI or unsuccessful and/ /or partial revascularization during the index PCI; (d) underwent PCI without stent implantation or with stents other than the EES, ZES, or BES; (e) underwent $\mathrm{CABG}$ as a revascularization strategy; (f) underwent thrombolysis; and (g) received overlap implantations of two or three types of EES, ZES, or BES. After excluding 3,222 patients, a total of 1,470 patients were included in the study. These patients were classified into three groups as follows: (a) AMI-RI patients undergoing PCI with EES implantation ( $\mathrm{n}=816$ ), (b) AMI-RI patients undergoing PCI with ZES implantation $(n=345)$, and (c) AMI-RI patients undergoing PCI with BES implantation ( $\mathrm{n}=309$ ) (Fig. 1). Follow-up data of these patients were obtained mainly through regularly scheduled outpatient visits. The present study was conducted according to the ethical principles of the Declaration of Helsinki, the best-known policy statement of the World Medical Association, which was revised in 2013 [16]. Similarly, the study protocol of the KAMIR-NIH registry was also approved by the ethics committee of each participating center [17]. Written informed consent was secured from all participants.

\section{Definition and clinical endpoints}

Kidney function was determined by the creatinine clearance $(\mathrm{CrCl})$ calculated using the Cockcroft-Gault formula [18], and it was based on the serum creatinine level upon admission. In this study, renal impairment was determined as $\mathrm{CrCl}<60 \mathrm{~mL} / \mathrm{min} / 1.73 \mathrm{~m}^{2}$ based on the serum creatinine level at the time of admission.

Acute myocardial infarction was defined according to current guidelines $[19,20]$, which include the typical rise and/or fall of biochemical markers of myocardial necrosis with at least one of the following: (a) clinical symptoms indicative of myocardial ischemia, (b) development of pathological Q-waves 


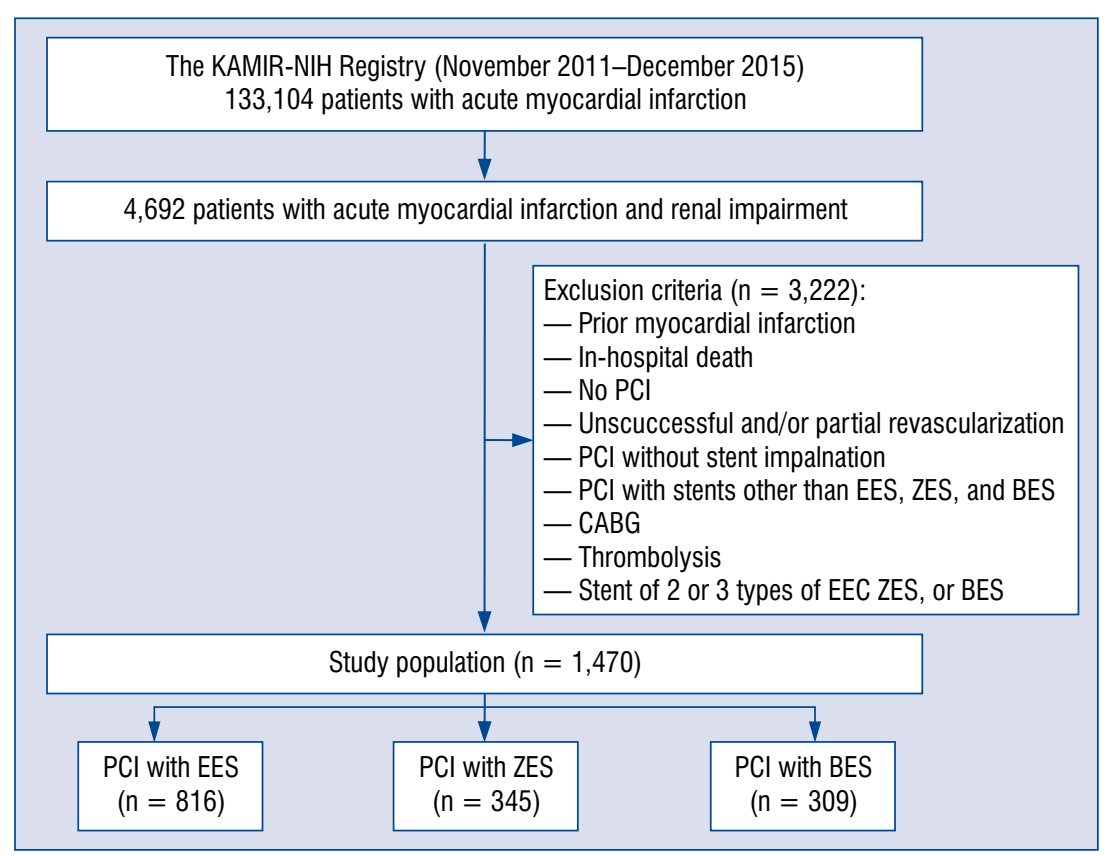

Figure 1. Flow chart for the selection of study participants; BES - biolimus-eluting stent; CABG - coronary artery bypass graft; EES - everolimus-eluting stent; KAMIR-NIH — Korea Acute Myocardial Infarction Registry-National Institutes of Health; $\mathrm{PCl}$ - percutaneous coronary intervention; ZES - zotarolimus-eluting stent.

in the 12-lead electrocardiogram (ECG) results, (c) ECG changes indicative of ischemia (elevation or depression of the ST-segment), and (d) imaging modalities suggesting MI (i.e., new loss of viable myocardium or new-onset regional wall motion abnormality). STEMI was defined as AMI with new-onset ST-segment elevation of at least $1 \mathrm{~mm}$ $(0.1 \mathrm{mV})$ in 2 or more contiguous leads, or new-onset left bundle branch block observed on ECG [21]. To quantitatively evaluate the left ventricle, left ventricular ejection fraction (LVEF) was examined using 2-dimensional echocardiography. The Killip classification, introduced in 1967, is defined as follows: Killip class I, no chronic heart failure; Killip class II, third heart sound and rales; Killip class III, overt pulmonary edema; and Killip class IV, cardiogenic shock [22]. Significant stenosis of the left main coronary artery (LMCA) was defined as an - at least $-50 \%$ reduction in the intraluminal diameter of the LMCA. Unprotected left main disease was defined as the presence of significant stenosis in the LMCA with no patent bypass graft to the left anterior descending coronary artery or left circumflex coronary artery. LMCA complex lesions were defined as the presence of significant stenosis in the LMCA with the presence of added epicardial coronary artery stenosis. Significant stenosis of other epicardial coronary arteries was defined as a reduction of at least $70 \%$ in the intraluminal diameter of the epicardial coronary artery. The degree of coronary flow was quantitatively classified according to the Thrombolysis In Myocardial Infarction (TIMI) flow grade.

Clinical follow-up was performed after the commencement of the study. The primary endpoint was major adverse cardiac and cerebrovascular events (MACCE), defined as the composite of all-cause death (cardiac and non-cardiac death), non-fatal MI, cerebrovascular accident (CVA), any revascularization using PCI or CABG, rehospitalization, and stent thrombosis. The secondary endpoints were net adverse clinical events (NACE), all-cause mortality, non-fatal MI, any revascularization, CVA, rehospitalization, and stent thrombosis. NACE was defined as a composite of all-cause death, non-fatal MI, and any revascularization. Any revascularization was defined as any repeat PCI or CABG of any part of the epicardial coronary arteries overall. Rehospitalization was defined as post-index admission due to angina and/ /or heart failure.

\section{Statistical analysis}

All data analysis was performed using both STATA version 15.0 (StataCorp, College Station, Texas, United States of America) and SPSS ver- 
sion 25.0 (SPSS Inc., Armonk, New York, USA). Continuous variables were expressed as means \pm standard deviation and analyzed using the Student t-test and the analysis of variance test. Discrete (categorical) variables were represented as percentages with numbers and analyzed using the Pearson chi-squared test, the Fisher two-by-two exact test, or linear by linear association. All results were considered statistically significant at $\mathrm{p}<0.05$.

To control for differences in baseline characteristics and potential confounding factors, the propensity score weighting method, called the inverse probability of treatment weighting (IPTW), was applied [23]. The propensity score was constructed by a multiple logistic regression model using a total of 41 covariates. Participants with missing data in these covariates or whose follow-up period after hospital discharge was estimated as 0 days were excluded from IPTW adjustment.

Unadjusted and IPTW-adjusted survival analyses were performed using the Kaplan-Meier analysis to determine the incidence of clinical outcomes, and log-rank (Mantel-Cox) tests were performed to evaluate differences among the treatment groups (i.e., EES, ZES, and BES groups).

\section{Results}

\section{Baseline clinical and procedural characteristics}

Baseline clinical characteristics are summarized in Table 1. Before IPTW adjustment, a significant baseline difference was observed between the three groups in terms of the Killip classification at admission. Although the proportion of Killip classification $>2$ in the ZES group was similar to that in the EES group, the BES group had a lower Killip classification than the other two groups. For discharge medications, there was a significant difference in the use of beta-blockers. The BES group received a relatively low prescription of this medication compared with the EES group. Although the EES group had a higher proportion of patients with DAPT $\geq 12$ months than the ZES group, the net difference was similar between the three groups.

In coronary angiography and procedural characteristics (Table 2), some differences were observed between the three groups. The BES group had a relatively lower incidence of pre-procedural TIMI flow grade $0-I$ and stent number $\geq 3$. The incidence of thrombus aspiration was higher in the ZES group than in the EES group. The incidence of RCA PCI was higher in the ZES group than in the other two groups. The ZES group had a higher proportion of STEMI patients compared to the EES group. Nonetheless, the net difference between the three groups was similar for these variables. Meanwhile, the overall difference was found in terms of the American Heart Association and the American College of Cardiology lesion type, vascular approach, staged PCI, LMCA complex lesions, LMCA PCI, stent number, total stent length, and total stent length $>60 \mathrm{~mm}$.

After IPTW adjustment, baseline clinical and procedural characteristics were balanced between the three DES groups (Suppl. Tables 1, 2).

\section{Long-term follow-up clinical outcomes}

After hospital discharge, follow-up was conducted with a median delay of 1,088 days. Clinical outcomes of MACCE, NACE, all-cause mortality, non-fatal MI, any revascularization, CVA, rehospitalization and stent thrombosis were determined. Kaplan-Meier analysis was performed to describe the crude (unadjusted) and IPTW-adjusted survival curves, and the pair-wise log-rank test results for these comparisons are shown in Figures 2 and 3. Before IPTW adjustment, there were no significant differences in any clinical outcomes between the three DES groups. However, after IPTW, a significant difference was found between these groups in terms of non-fatal MI. In the ZES group, the incidence of non-fatal MI was higher than in the other two groups. The number of patients at risk is shown in Table 3.

\section{Discussion}

This study demonstrates that except for non-fatal MI, there was no significant difference among the three stent groups concerning long-term MACCE, NACE, all-cause mortality, any revascularization, CVA, rehospitalization and stent thrombosis. Regarding non-fatal MI, despite the significant difference not derived from unadjusted raw data analysis, IPTW-adjusted analysis showed that the ZES group had a higher incidence of nonfatal MI than the other two groups ( $p=0.005)$.

Chronic kidney disease is a major health issue, with an increasing prevalence worldwide [24]; similarly, it is a debilitating medical condition, culminating in ESKD requiring dialysis or kidney transplantation, and is recognized as an independent cardiovascular risk factor $[2,25]$. Among patients with renal impairment, cardiovascular events such as $\mathrm{CAD}$, are the main cause of mortality $[25,26]$. CKD 


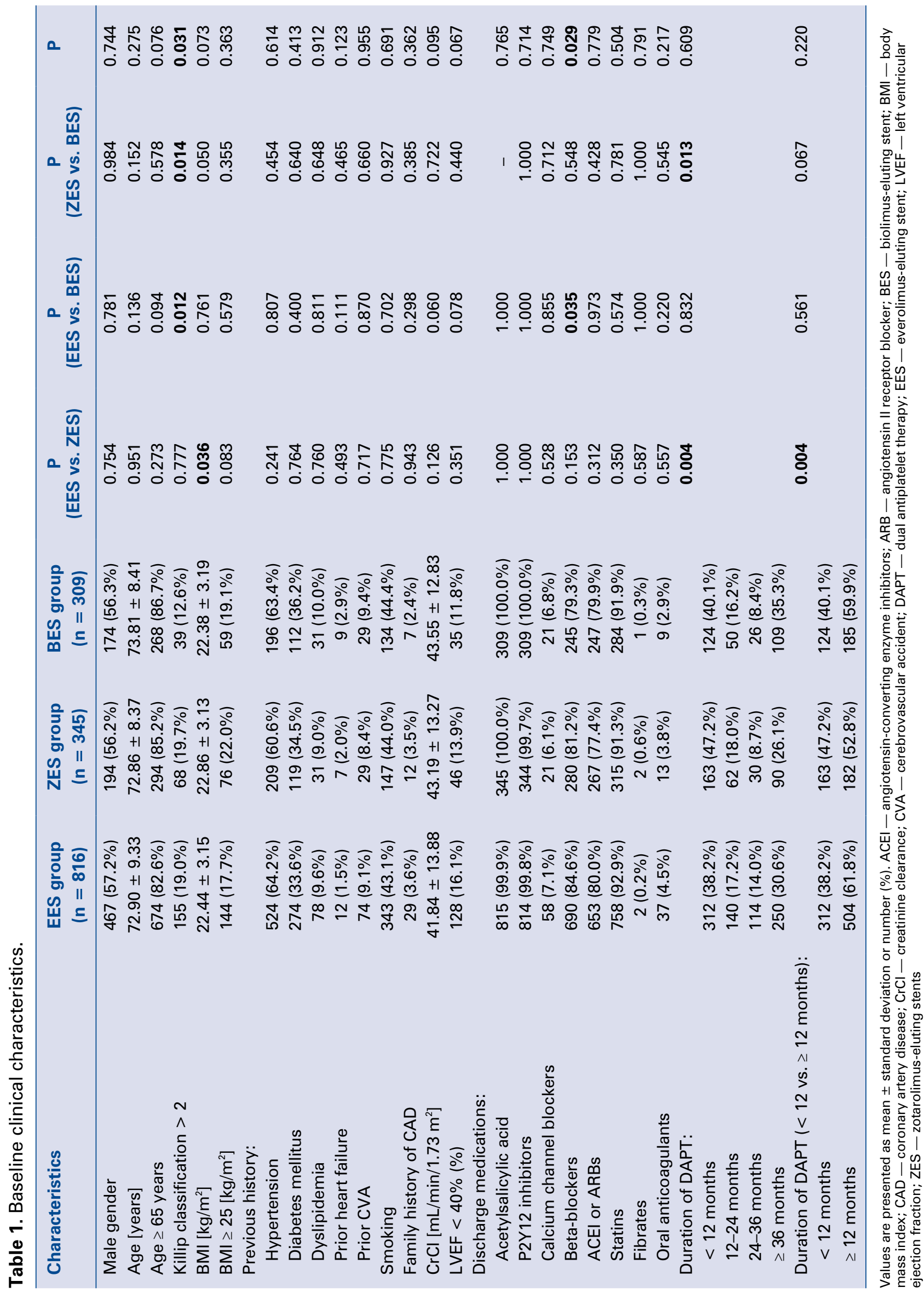




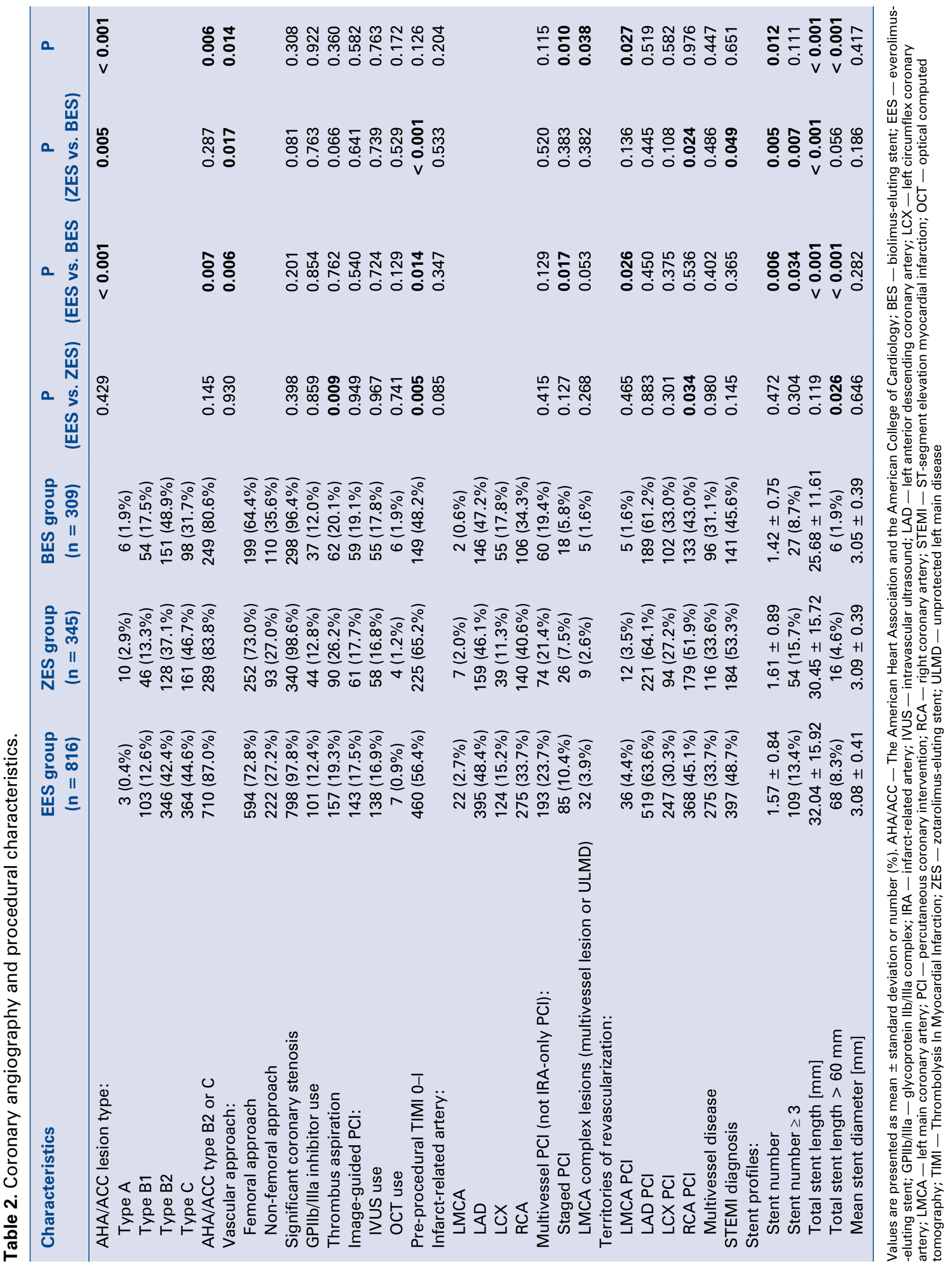




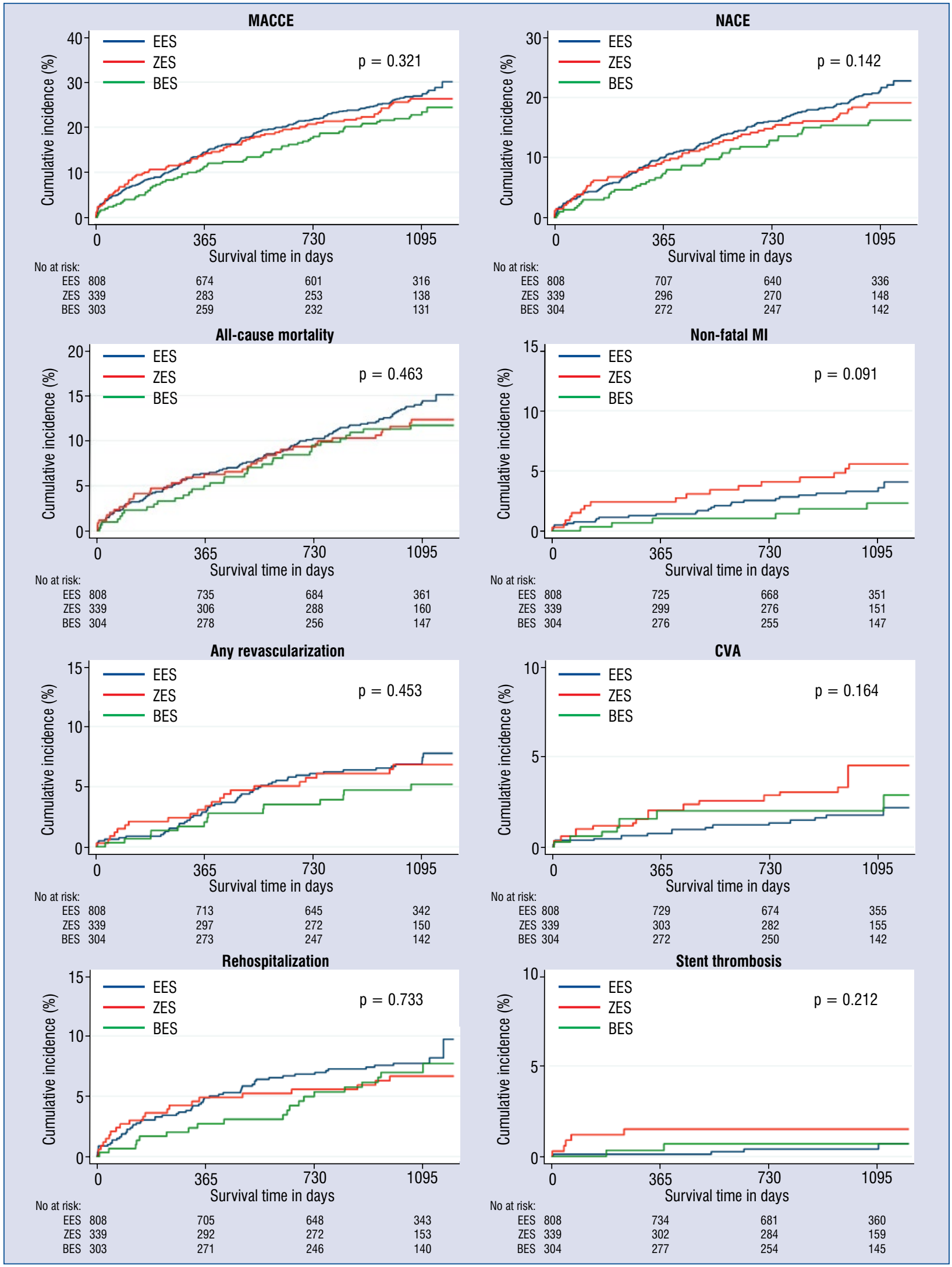

Figure 2. Kaplan-Meier survival analyses of long-term follow-up clinical outcomes (MACCE, NACE, and all-cause mortality, non-fatal MI, any revascularization, CVA, rehospitalization, and stent thrombosis), stratified according to stent types (before inverse probability of treatment weighting); CVA - cerebrovascular accident; MACCE — major adverse cardiac and cerebrovascular events; MI — myocardial infarction; NACE — net adverse clinical events. 


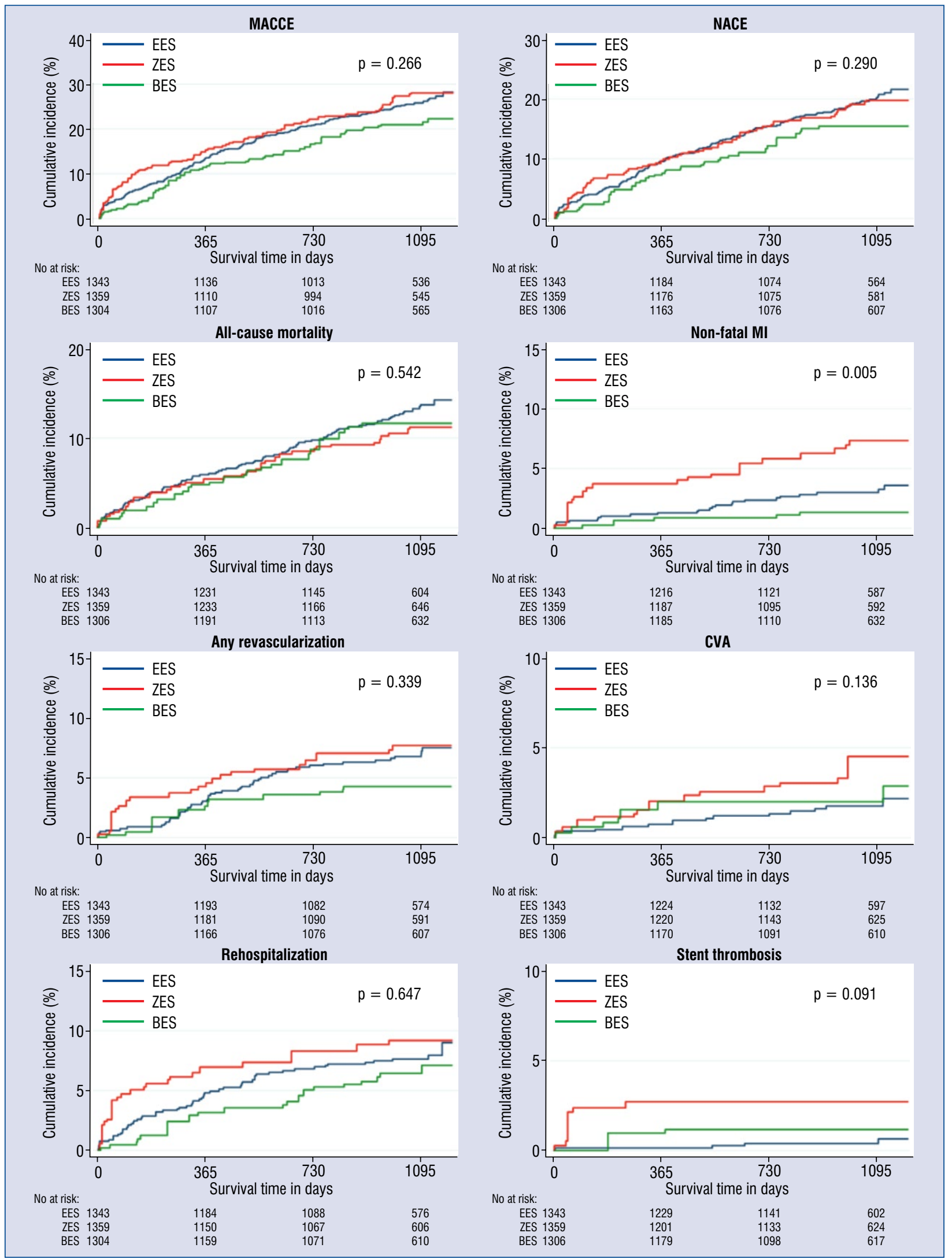

Figure 3. Kaplan-Meier survival analyses of long-term follow-up clinical outcomes (MACCE, NACE, and all-cause mortality, non-fatal MI, any revascularization, CVA, rehospitalization, and stent thrombosis), stratified according to stent types (after inverse probability of treatment weighting); CVA - cerebrovascular accident; MACCE — major adverse cardiac and cerebrovascular events; $\mathrm{MI}$ - myocardial infarction; NACE - net adverse clinical events. 
patients tend to have a higher risk of experiencing cardiovascular events than patients with normal kidney function [27, 28]. Furthermore, the 2-year mortality rate after AMI is approximately $50 \%$ in ESKD patients, which is much higher than the mortality rate after AMI in the general population [29]. Some large-scale studies demonstrated that reduced kidney function was independently associated with an increased risk of mortality and cardiovascular events in patients with reduced LVEF [30, 31]. A similar trend was observed between kidney function and cardiovascular events in an AMI setting [25].

Although the mechanism underlying the development of cardiovascular disorders by renal impairment is still not well understood, it may be explained by several factors related to renal impairment. The progression of renal impairment is closely related to systemic inflammation and oxidative stress, which are responsible for the clinical manifestations of numerous complications, including atherosclerosis, vascular calcification (calciphylaxis), anemia, heart failure, and derangements in calcium-phosphate homeostasis (mineral and bone disorders) [32-34]. Additionally, CKD is associated with an increased risk of thrombosis [35]. In CKD patients, clinically relevant thrombosis often presents as venous thromboembolism, vascular access-associated thrombosis, and right atrial thrombosis [35]. Similarly, thrombosis may occur within arteries, presenting as CAD, CVA, or peripheral artery disease [36]. These factors are directly and/or indirectly associated with cardiovascular disorders and may contribute to the development of cardiovascular events in patients with renal impairment. Meanwhile, the prevalence of coronary risk factors tends to be high in CKD patients $[37,38]$. In the present study, the proportion of patients with hypertension and diabetes mellitus increased with the worsening of the $\mathrm{CrCl}$ (Suppl. Table 3). Because these coronary risk factors, including hypertension and diabetes mellitus, are equally recognized as predictors of renal impairment, they may worsen kidney function, subsequently increasing the influence of such risk factors [25]. This synergistic effect is also reflected in the present study, as lower $\mathrm{CrCl}$ caused lower LVEF with increasing incidence of the two aforementioned coronary risk factors (Suppl. Table 3).

Several clinical studies have compared the clinical outcomes of implanted coronary stents in patients with AMI who underwent PCI. DES implantation showed better clinical outcomes than the bare-metal stent in reducing MI and mortality after PCI [39, 40]. Some studies compared 2G-DES and BES in AMI patients. Kim et al. [41] compared the 2-year clinical outcomes of 2 G-DES with those of BES in AMI patients with dyslipidemia after PCI and found similar results. Choe et al. [42] reported that BES shows clinical outcomes similar to those of new-generation DES. An article about the network meta-analysis of the efficacy and safety of coronary stents in patients with STEMI showed comparable results regarding the risk of primary outcomes between the DES groups, including the ZES, EES, and BES groups [40].

Similarly, there are published papers comparing stents in patients with AMI and renal impairment. Hachinohe et al. [43] reported that ZES results in a higher frequency of major adverse cardiovascular events (MACE) due to the increased TLR rate compared with SES in AMI patients with concomitant CKD. Ahmed et al. [44] compared the ZES and EES in STEMI patients with CKD undergoing PCI, and their results showed similarities with the risk of 12-month MACE and death in patients with STEMI and CKD undergoing PCI.

Unlike these studies comparing two stent groups among AMI-RI patients, the current study is the first to compare clinical outcomes between three DES groups in selected AMI-RI patients undergoing PCI. This study highlights that the use of ZES is associated with the occurrence of non-fatal MI compared with the use of the other two DESs. In addition, clinical findings herein, were based on a longer follow-up period than in previously published articles that were mentioned earlier.

Nonetheless, it is still unclear why this significant result was derived regarding non-fatal MI. It was mainly driven by the difference between ZES and EES groups, or between ZES and EES groups. In a comparative study evaluating 5-year efficacy of both EES and Resolute ZES in PCI-treated ACS patients, Resolute ZES demonstrated worse long-term outcomes than EES [45]. The authors of this study emphasized that the clinical differences between the two stent types were mainly driven by the polymer characteristics, not by the antiproliferative agents. Because the fluoropolymer, a highly fluorinated bilayer copolymer, coated with EES platform has high biocompatibility, reduces platelet adhesion and thrombus formation, these characteristics seems to influence better long-term outcomes in EES compared to Resolute ZES. Meanwhile, unlike both ZES and EES, which have durable polymers, BES has biodegradable polymers. In the BIOSTEMI trial, biodegradable polymer DES was statistically superior to durable polymer DES among STEMI patients [46]. Similarly, in the present study, BES showed relatively 


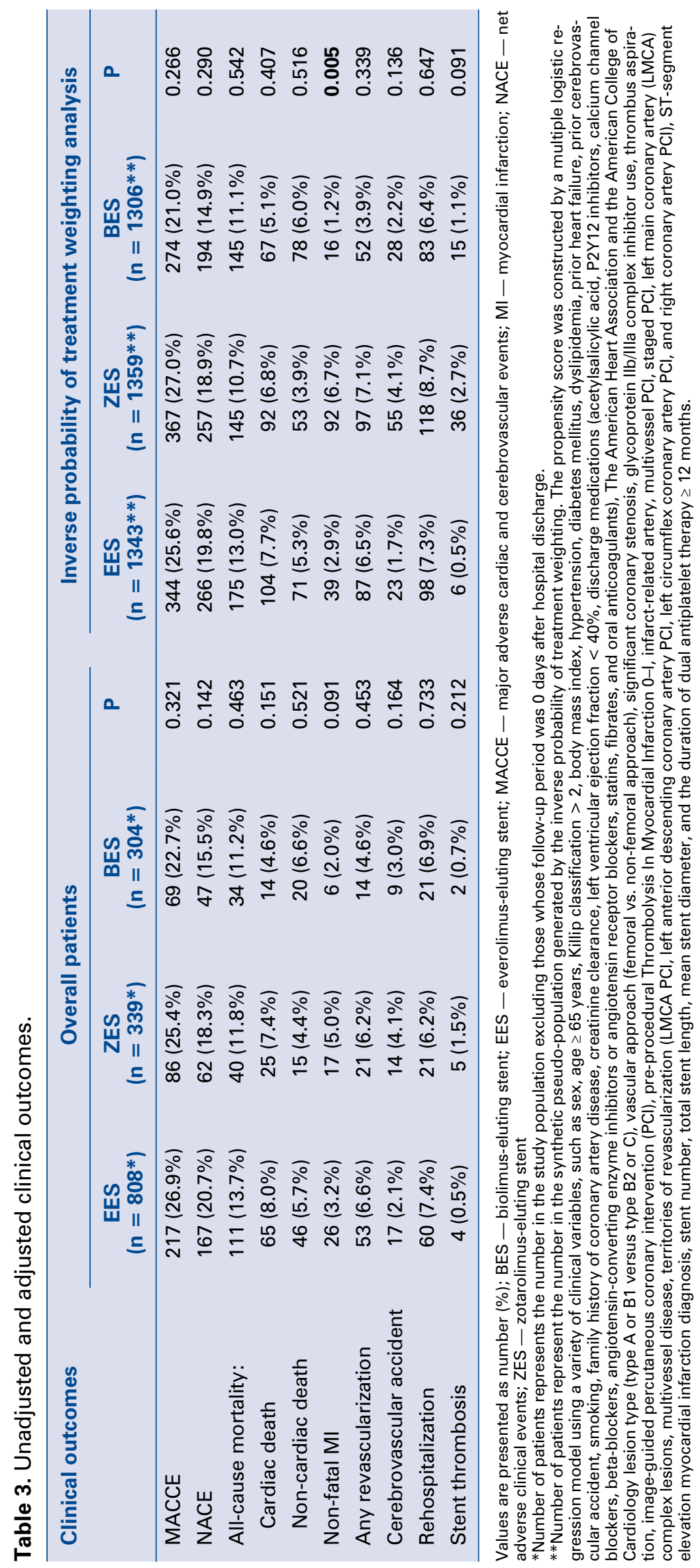


good clinical outcomes (MACCE, NACE, cardiac death, non-fatal MI, any revascularization, and rehospitalization), although many of them were not statistically insignificant. Additionally, the statistical process should be considered in interpreting these results. In total, 110 patients (patients with a follow-up of 0 days or patients with any missing value in 41 covariates) were excluded from the IPTW-adjusted analysis. Thus, selection bias may have occurred in this process, causing disparities in non-fatal MI outcomes before and after IPTW (p-value of 0.091 before IPTW, and 0.005 after IPTW).

\section{Limitations of the study}

There are several limitations to be considered when interpreting the results of this study. First, the contributing institutions in the KAMIR-NIH registry tended to be tertiary centers with a higher volume of patients than average medical institutes. Thus, the mortality rates and treatment practice patterns could not be generalized to all medical institutions treating STEMI patients. Second, the information concerning hemodialysis in the KAMIR-NIH registry was not considered, making it impossible to separate hemodialysis patients from non-hemodialysis patients. Third, detailed stent information such as stent material, stent linker type, strut thickness, and polymer coating, to account for the heterogeneity of each DES, were not included in the analysis. Moreover, the KAMIR-NIH registry does not include several important angiographic profiles and lesion characteristics such as the presence of bifurcation lesion, chronic total occlusion, overlapping stents, use of shockwave intravascular lithotripsy and the use of rotational atherectomy. Fourth, considering the timing of data collection, older types of DESs, which are no longer used in routine clinical practice, could undoubtedly also be included in the analysis. Fifth, this study was based on an observational registry; however, it was a non-randomized study. Hence, although statistical adjustment using the propensity score weighting method was conducted to overcome this limitation, a large-scale multicenter randomized controlled trial is needed in the future.

\section{Conclusions}

In summary, there were no differences in the long-term clinical outcomes between the ZES, EES, and BES groups in AMI-RI patients undergoing PCI, except for non-fatal MI. Unlike EES, ZES may be a predictor of non-fatal MI.

\section{Acknowledgments}

This study was supported by grants from the Korean Health Technology R \& D Project, Ministry of Health \& Welfare (HI13C1527), and the Research of Korea Centers for Disease Control and Prevention (2016-ER6304-01), Republic of Korea.

\section{Conflict of interest: None declared}

\section{References}

1. Debella YT, Giduma HD, Light RP, et al. Chronic kidney disease as a coronary disease equivalent--a comparison with diabetes over a decade. Clin J Am Soc Nephrol. 2011; 6(6): 1385-1392, doi: 10.2215/CJN.10271110, indexed in Pubmed: 21393492.

2. Go AS, Chertow GM, Fan D, et al. Chronic kidney disease and the risks of death, cardiovascular events, and hospitalization. N Engl J Med. 2004; 351(13): 1296-1305, doi: 10.1056/NEJMoa041031, indexed in Pubmed: 15385656.

3. de Jager DJ, Grootendorst DC, Jager KJ, et al. Cardiovascular and noncardiovascular mortality among patients starting dialysis. JAMA. 2009; 302(16): 1782-1789, doi: 10.1001/jama.2009.1488, indexed in Pubmed: 19861670.

4. Sarnak MJ, Levey AS. Epidemiology, diagnosis, and management of cardiac disease in chronic renal disease. J Thromb Thrombolysis. 2000; 10(2): 169-180, doi: 10.1023/a:1018718727634, indexed in Pubmed: 11005939.

5. Fischman DL, Leon MB, Baim DS, et al. A randomized comparison of coronary-stent placement and balloon angioplasty in the treatment of coronary artery disease. Stent Restenosis Study Investigators. N Engl J Med. 1994; 331(8): 496-501, doi: 10.1056/ NEJM199408253310802, indexed in Pubmed: 8041414.

6. Serruys PW, de Jaegere P, Kiemeneij F, et al. A comparison of balloon-expandable-stent implantation with balloon angioplasty in patients with coronary artery disease. Benestent Study Group. N Engl J Med. 1994; 331(8): 489-495, doi: 10.1056/ NEJM199408253310801, indexed in Pubmed: 8041413.

7. Moses JW, Leon MB, Popma JJ, et al. SIRIUS Investigators. Sirolimus-eluting stents versus standard stents in patients with stenosis in a native coronary artery. N Engl J Med. 2003; 349(14): 1315-1323, doi: 10.1056/NEJMoa035071, indexed in Pubmed: 14523139.

8. Stone GW, Ellis SG, Cox DA, et al. A polymer-based, paclitaxeleluting stent in patients with coronary artery disease. $\mathrm{N} \mathrm{Engl}$ J Med. 2004; 350(3): 221-231, doi: 10.1056/NEJMoa032441, indexed in Pubmed: 14724301.

9. Virmani R, Guagliumi G, Farb A, et al. Localized hypersensitivity and late coronary thrombosis secondary to a sirolimus-eluting stent: should we be cautious? Circulation. 2004; 109(6): 701-705, doi: 10.1161/01.CIR.0000116202.41966.D4, indexed in Pubmed: 14744976.

10. Kang SH, Chae IH, Park JJ, et al. Stent thrombosis with drugeluting stents and bioresorbable scaffolds: evidence from a network meta-analysis of 147 trials. JACC Cardiovasc Interv. 2016; 9(12): 1203-1212, doi: 10.1016/j.jcin.2016.03.038, indexed in Pubmed: 27262860.

11. Palmerini T, Biondi-Zoccai G, Della Riva D, et al. Stent thrombosis with drug-eluting stents: is the paradigm shifting? J Am Coll Cardiol. 2013; 62(21): 1915-1921, doi: 10.1016/j.jacc.2013.08.725, indexed in Pubmed: 24036025. 


\section{Cardiology Journal}

12. Serruys PW, Farooq V, Kalesan B, et al. Improved safety and reduction in stent thrombosis associated with biodegradable polymer-based biolimus-eluting stents versus durable polymerbased sirolimus-eluting stents in patients with coronary artery disease: final 5-year report of the LEADERS (Limus Eluted From A Durable Versus ERodable Stent Coating) randomized, noninferiority trial. JACC Cardiovasc Interv. 2013; 6(8): 777-789, doi: 10.1016/j.jcin.2013.04.011, indexed in Pubmed: 23968698.

13. Natsuaki M, Kozuma K, Morimoto T, et al. Final 3-Year Outcome of a Randomized Trial Comparing Second-Generation Drug-Eluting Stents Using Either Biodegradable Polymer or Durable Polymer: NOBORI Biolimus-Eluting Versus XIENCE/ /PROMUS Everolimus-Eluting Stent Trial. Circ Cardiovasc Interv. 2015; 8(10): 815-818, doi: 10.1161/CIRCINTERVENTIONS.115.002817, indexed in Pubmed: 26446596.

14. Vlachojannis GJ, Smits PC, Hofma SH, et al. Long-term clinical outcomes of biodegradable polymer biolimus-eluting stents versus durable polymer everolimus-eluting stents in patients with coronary artery disease: three-year follow-up of the COMPARE II (Abluminal biodegradable polymer biolimus-eluting stent versus durable polymer everolimus-eluting stent) trial. EuroIntervention. 2015; 11(3): 272-279, doi: 10.4244/EIJV11I3A53, indexed in Pubmed: 26196753.

15. Matsushita K, van der Velde M, Astor BC, et al. Association of estimated glomerular filtration rate and albuminuria with allcause and cardiovascular mortality in general population cohorts: a collaborative meta-analysis. Lancet. 2010; 375(9731): 2073-2081, doi: 10.1016/S0140-6736(10)60674-5, indexed in Pubmed: 20483451.

16. World Medical Association Declaration of Helsinki: ethical principles for medical research involving human subjects. JAMA. 2013; 310(20): 2191, doi: 10.1001/jama.2013.281053.

17. Kim JuH, Chae SC, Oh DJ, et al. Multicenter Cohort Study of Acute Myocardial Infarction in Korea-Interim Analysis of the Korea Acute Myocardial Infarction Registry-National Institutes of Health Registry. Circ J. 2016; 80(6): 1427-1436, doi: 10.1253/ circj.CJ-16-0061, indexed in Pubmed: 27118621.

18. Cockcroft DW, Gault MH. Prediction of creatinine clearance from serum creatinine. Nephron. 1976; 16(1): 31-41, doi: 10.1159/000180580, indexed in Pubmed: 1244564.

19. Neumann FJ, Sousa-Uva M, Ahlsson A, et al. 2018 ESC/EACTS Guidelines on myocardial revascularization. Eur Heart J. 2019; 40: 87-165, doi: 10.1093/eurheartj/ehy394, indexed in Pubmed: 30165437.

20. Roffi M, Patrono C, Collet JP, et al. 2015 ESC Guidelines for the management of acute coronary syndromes in patients presenting without persistent ST-segment elevation. Eur Heart J. 2015; 37(3): 267-315, doi: 10.1093/eurheartj/ehv320.

21. Ibanez B, James S, Agewall S, et al. 2017 ESC Guidelines for the management of acute myocardial infarction in patients presenting with ST-segment elevation: The Task Force for the management of acute myocardial infarction in patients presenting with ST-segment elevation of the European Society of Cardiology (ESC). Eur Heart J. 2018; 39: 119-77, doi: 10.1093/eurheartj/ ehx393, indexed in Pubmed: 28886621.

22. Killip T, Kimball J. Treatment of myocardial infarction in a coronary care unit. Am J Cardiol. 1967; 20(4): 457-464, doi: 10.1016/0002-9149(67)90023-9.

23. Yoshida K, Hernández-Díaz S, Solomon DH, et al. Matching Weights to Simultaneously Compare Three Treatment Groups: Comparison to Three-way Matching. Epidemiology. 2017; 28(3):
387-395, doi: 10.1097/EDE.0000000000000627, indexed in Pubmed: 28151746.

24. Jha V, Garcia-Garcia G, Iseki K, et al. Chronic kidney disease: global dimension and perspectives. Lancet. 2013; 382(9888): 260-272, doi: 10.1016/S0140-6736(13)60687-X, indexed in Pubmed: 23727169.

25. Anavekar NS, McMurray JJV, Velazquez EJ, et al. Relation between renal dysfunction and cardiovascular outcomes after myocardial infarction. N Engl J Med. 2004; 351(13): 1285-1295, doi: 10.1056/NEJMoa041365, indexed in Pubmed: 15385655.

26. Foley RN, Murray AM, Li S, et al. Chronic kidney disease and the risk for cardiovascular disease, renal replacement, and death in the United States Medicare population, 1998 to 1999. J Am Soc Nephrol. 2005; 16(2): 489-495, doi: 10.1681/ASN.2004030203, indexed in Pubmed: 15590763.

27. Al Suwaidi J, Reddan DN, Williams K, et al. Prognostic implications of abnormalities in renal function in patients with acute coronary syndromes. Circulation. 2002; 106(8): 974-980, doi: 10.1161/01. cir.0000027560.41358.b3, indexed in Pubmed: 12186803.

28. Wright RS, Reeder GS, Herzog CA, et al. Acute myocardial infarction and renal dysfunction: a high-risk combination. Ann Intern Med. 2002; 137(7): 563-570, doi: 10.7326/0003-4819-1377-200210010-00007, indexed in Pubmed: 12353943.

29. Sarnak MJ, Levey AS, Schoolwerth AC, et al. Kidney disease as a risk factor for development of cardiovascular disease: a statement from the American Heart Association Councils on Kidney in Cardiovascular Disease, High Blood Pressure Research, Clinical Cardiology, and Epidemiology and Prevention. Hypertension. 2003; 42(5): 1050-1065, doi: 10.1161/01. HYP.0000102971.85504.7c, indexed in Pubmed: 14604997.

30. Freeman RV, Mehta RH, Al Badr W, et al. Influence of concurrent renal dysfunction on outcomes of patients with acute coronary syndromes and implications of the use of glycoprotein IIb/IIIa inhibitors. J Am Coll Cardiol. 2003; 41(5): 718-724, doi: 10.1016/ s0735-1097(02)02956-x, indexed in Pubmed: 12628712.

31. Sørensen CR, Brendorp B, Rask-Madsen C, et al. The prognostic importance of creatinine clearance after acute myocardial infarction. Eur Heart J. 2002; 23(12): 948-952, doi: 10.1053/ euhj.2001.2989, indexed in Pubmed: 12069449.

32. Gupta J, Mitra N, Kanetsky PA, et al. CRIC Study Investigators. Association between albuminuria, kidney function, and inflammatory biomarker profile in CKD in CRIC. Clin J Am Soc Nephrol. 2012; 7(12): 1938-1946, doi: 10.2215/CJN.03500412, indexed in Pubmed: 23024164.

33. Shlipak MG, Fried LF, Crump C, et al. Elevations of inflammatory and procoagulant biomarkers in elderly persons with renal insufficiency. Circulation. 2003; 107(1): 87-92, doi: 10.1161/01. cir.0000042700.48769.59, indexed in Pubmed: 12515748.

34. Stenvinkel P, Heimbürger O, Paultre F, et al. Strong association between malnutrition, inflammation, and atherosclerosis in chronic renal failure. Kidney Int. 1999; 55(5): 1899-1911, doi: 10.1046/j.1523-1755.1999.00422.x, indexed in Pubmed: 10231453.

35. Lutz J, Menke J, Sollinger D, et al. Haemostasis in chronic kidney disease. Nephrol Dial Transplant. 2014; 29(1): 29-40, doi: 10.1093/ndt/gft209, indexed in Pubmed: 24132242.

36. Casserly L, Dember L. Thrombosis in end-stage renal disease. Semin Dial. 2003; 16(3): 245-256, doi: 10.1046/j.1525139x.2003.16048.x.

37. Luft FC. Renal disease as a risk factor for cardiovascular disease. Basic Res Cardiol. 2000; 95 Suppl 1: I72-I76, doi: 10.1007/ s003950070013, indexed in Pubmed: 11192357. 
38. National Kidney Fundation. K/DOQI clinical practice guidelines for chronic kidney disease: evaluation, classification, and stratification. Am J Kidney Dis. 2002; 39: S1-S266, indexed in Pubmed: 11904577.

39. Sung SH, Chen TC, Cheng HM, et al. Comparison of clinical outcomes in patients undergoing coronary intervention with drug-eluting stents or bare-metal stents: a Nationwide Population Study. Acta Cardiol Sin. 2017; 33(1): 10-19, doi: 10.6515/ acs20160608a, indexed in Pubmed: 28115802.

40. Chichareon P, Modolo R, Collet C, et al. Efficacy and safety of stents in ST-segment elevation myocardial infarction. J Am Coll Cardiol. 2019; 74(21): 2572-2584, doi: 10.1016/j.jacc.2019.09.038, indexed in Pubmed: 31753202.

41. Kim YH, Her AY, Jeong MHo, et al. Two-year clinical outcomes of zotarolimus- and everolimus-eluting durable-polymer-coated stents versus biolimus-eluting biodegradable-polymer-coated stent in patients with acute myocardial infarction with dyslipidemia after percutaneous coronary intervention: data from the KAMIR. Heart Vessels. 2019; 34(2): 237-250, doi: 10.1007/ s00380-018-1251-0, indexed in Pubmed: 30167772.

42. Choe JC, Cha KS, Jang HY, et al. Korea Acute Myocardial Infarction Registry Investigators. Outcomes of acute myocardial infarction patients implanted with biodegradable polymer biolimus-eluting stents versus new-generation durable polymer drug-eluting stents: a retrospective analysis. Angiology. 2017; 68(8): 698-706, doi: 10.1177/0003319716679339, indexed in Pubmed: 27872316.

43. Hachinohe D, Jeong MHo, Saito S, et al. Korea Acute Myocardial Infarction Registry Investigators. Comparison of drug-eluting stents in acute myocardial infarction patients with chronic kidney disease. Korean J Intern Med. 2012; 27(4): 397-406, doi: 10.3904/kjim.2012.27.4.397, indexed in Pubmed: 23269880.

44. Ahmed K, Jeong MHo, Chakraborty R, et al. Other Korea Acute Myocardial Infarction Registry Investigators. Comparison of zotarolimus- and everolimus-eluting stents in patients with ST-elevation myocardial infarction and chronic kidney disease undergoing primary percutaneous coronary intervention. J Cardiol. 2014; 64(4): 273-278, doi: 10.1016/j.jjcc.2014.02.002, indexed in Pubmed: 24631465.

45. Koni E, Wanha W, Ratajczak J, et al. Five-year comparative efficacy of everolimus-eluting vs. Resolute zotarolimus-eluting stents in patients with acute coronary syndrome undergoing percutaneous coronary intervention. J Clin Med. 2021; 10(6), doi: 10.3390/jcm10061278, indexed in Pubmed: 33808678.

46. Iglesias JF, Muller O, Heg D, et al. Biodegradable polymer sirolimus-eluting stents versus durable polymer everolimus-eluting stents in patients with ST-segment elevation myocardial infarction (BIOSTEMI): a single-blind, prospective, randomised superiority trial. Lancet. 2019; 394(10205): 1243-1253, doi: 10.1016/ S0140-6736(19)31877-X, indexed in Pubmed: 31488372. 